\title{
Design and Implementation of Non - base Station Multi - level Communication Network Based on Link16
}

\author{
Junhao Ouyang ${ }^{1,}$, Shaojing $\mathrm{Su}^{1}$ \\ ${ }^{1}$ National University of Defense Technology, Hunan 410000, China; \\ aouyangjunhao1993@163.com,
}

Keywords: multi-level communication network, Link16, SDR.

\begin{abstract}
This paper introduces the establishment of multi-level communication network through wireless module based on link16 data link protocol in the absence of fixed center communication nodes such as base stations, thus realizing bidirectional transmission of communication front-end to control center data. Mainly using TDMA communication mechanism, by the control center to the communication node to send control commands, and in the fixed time slot to receive the corresponding data. Experiments show that when the communication node is controlled within a certain number, the communication system can get better communication effect, suitable for mine, field rescue and other non-base station under the conditions of communication.
\end{abstract}

\section{Introduction}

Battlefield information is very important, cannot be intercepted by hostile forces. Field operations, the establishment and maintenance of base stations spend huge, slow, great impact on the mobility of the troops [1].

The existence of the above problems, to a certain extent, affected the combat forces in the actual combat level of the upgrade. The link16 data link uses advanced direct sequence spread spectrum, frequency hopping, skip hybrid spread spectrum technology, RS error correction coding and interleaving techniques, which make the system stable and reliable, with strong anti-jamming performance[2,3]. It is necessary to study a detection and evaluation system which can simulate the realistic light weapon firing training environment and make the trainees close to the actual training, scientific, accurate and timely assessment of the battlefield situation.

\section{General design of system}

Construction of Communication System. The whole communication system is mainly composed of communication terminal, communication relay node and control center. The system structure is shown in Fig.

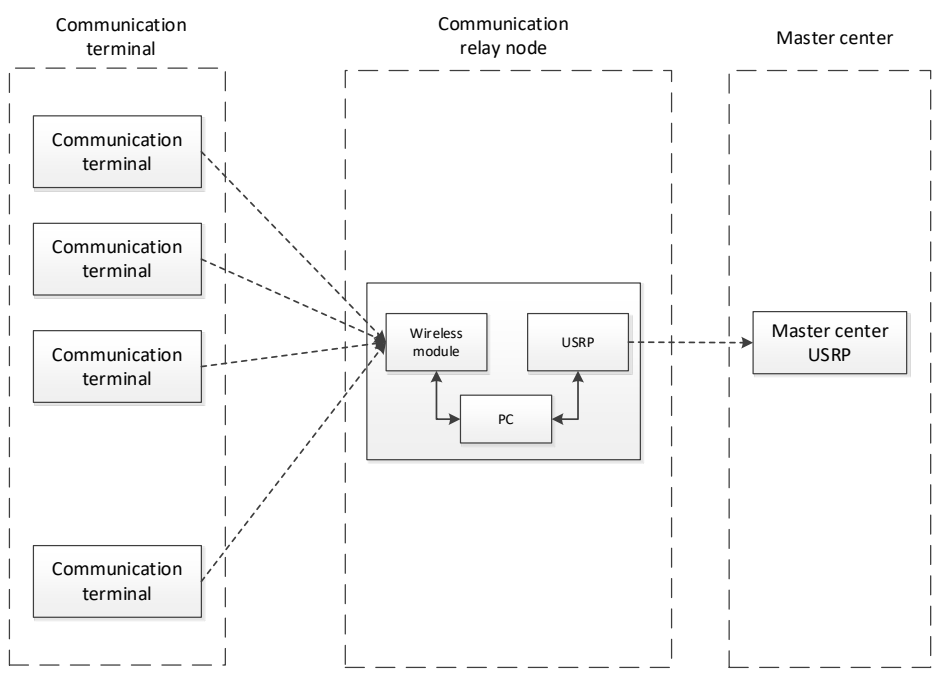

Fig. 1 System Architecture 
The communication terminal is mainly responsible for collecting terminal basic information and transmitting it to the communication relay node through the wireless communication module. The communication relay node is responsible for receiving and storing the data sent by the communication node (control center) and forwarded to the control center (communication node). The control center is composed of wireless communication module and database server. It is responsible for the establishment and maintenance of wireless communication networks, sending data and commands, receiving slave data and stored in the system database, statistical analysis results, etc., is the system of information exchanging bridge.

Communication relay node hardware. Communication relay node is a bridge in the communication system, responsible for communication between the communication terminal and the control center, because the software radio can be a good solution to the various standards and protocols are not compatible with each other, and the frequency allocation is inconsistent, is a multi-band, Multi-mode, programmable, versatile radio. Therefore, the communication relay node will use the wireless module and the software radio platform USRP work together, the wireless module collects the terminal data and sends the data to the control center through the USRP, and the control center communicates with the relay node through the USRP. Communication relay node consists of PC, wireless module YL-500IW, software radio platform USRP, battery and its peripheral circuit, as shown in Figure 2. The node receives the communication terminal data, after integration with the control center for data transmission.

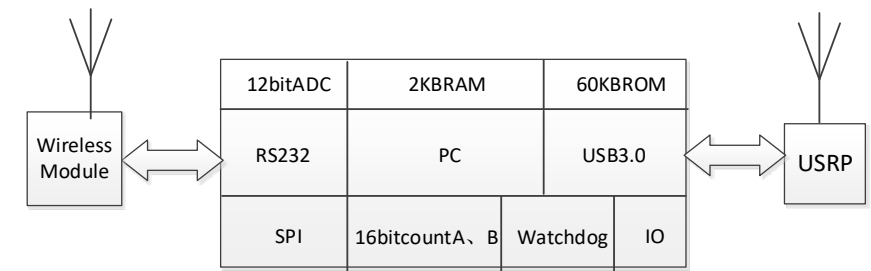

Fig. 2 Communication relay node hardware structure

Wireless Data Communication Protocol. JTIDS is the data terminal of link16 data link. It adopts $\mathrm{J}$ series message coding standard and operates in band $960 \sim 1215 \mathrm{MHz}$ [4]. It adopts synchronous time division multiplexing and multiple access network[5]. It has anti-interference with frequency hopping, direct sequence spread spectrum and hop the way. JTIDS system data link layer structure as shown in the figure3, the top branch of the transmitter part of the next branch for the receiver part[6].

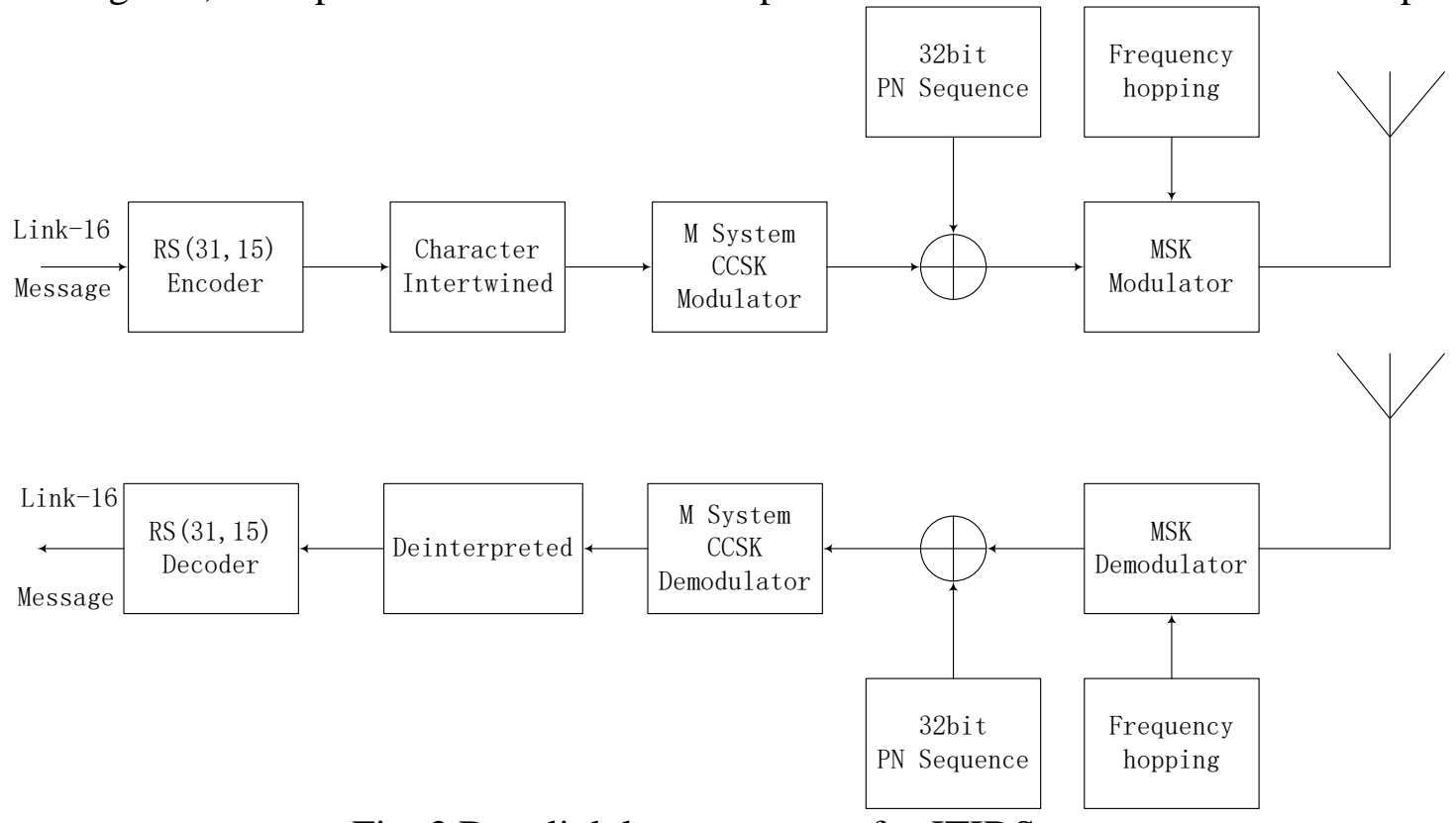

Fig. 3 Data link layer structure for JTIDS

Wireless network communication using master-slave mode, that is, each communication initiated by the host. Need to communicate with the slave, the host sends a data request frame to the slave, 
when the host query, the corresponding slave can work status, battery power, address and other status information to the host. The data transmitted by the wireless communication system includes control information and data information.

The communication process is shown in Figure 4.

(1) the master and slave power, the host is in the sending state, the slave is in the receiving state.

(2) the host sends a data request frame to the first slave, the transmission mode is converted to the receiving mode, and the timer starts to work. After receiving the host request frame, the slave mode changes from the receiving mode to the transmitting mode, To the host.

(3) If the host receives the data sent from the slave, the data is stored in the memory; if no data is received, the error counter is incremented. Until the timer ends.

(4) the address of the second slave into the host, and the host to launch mode.

(5) Go to the first step.

(6) When the upper control command frame, PC analysis control command frame, in the next polling slot, the control object address written to the host, while the control command word written to the host data request frame, send, host Reschedule from the first slave.

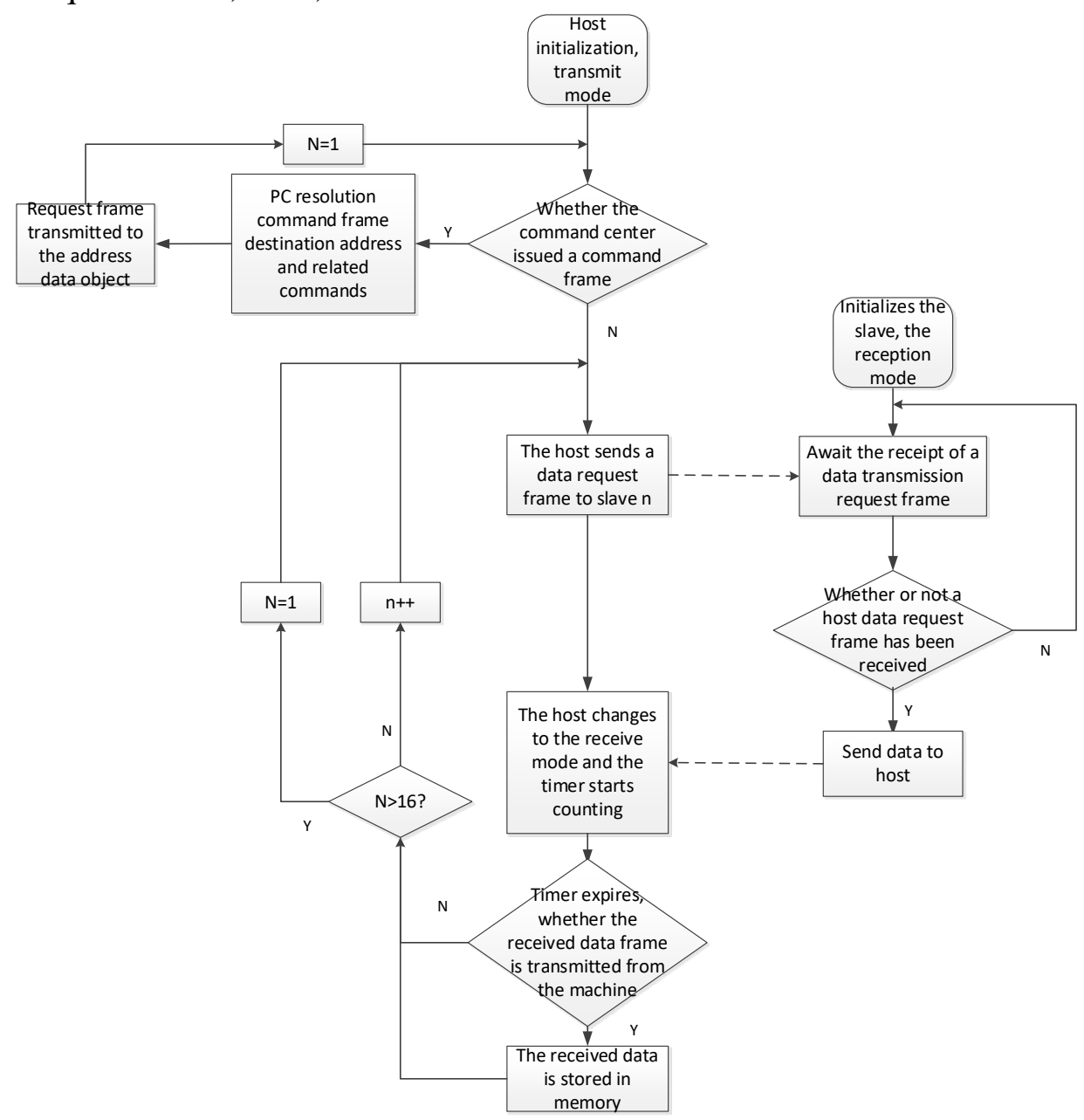

Fig. 4 Master and slave network establishment and communication flow chart

Principle of System. The communication terminal is responsible for collecting the terminal information. In the communication network, the communication relay node uses the time-division multiplexing communication mechanism and the communication terminal to establish the point-to-multipoint wireless communication network, and receives the communication terminal data, after data integration, Send the data to the control center. In the control center and the communication relay node network, the data is transmitted in link16 waveform. The control center communicates with the host as a host control network, receives the data sent by the communication relay node, and stores it in the database server for recording statistics and processing. After the control center completes the evaluation, the processing result and the control information are fed back to the 
communication relay node. The relay node receives the control center data and forwards it to the corresponding communication terminal and performs the specific operation.

System Test. This system is mainly to achieve the functional data transmission between the communication terminal and the control center. Wireless module baud rate is set to 9600,8 data bits, 1 stop bit, no check bit, control center by the end of the PC uses serial debugging assistant instead of testing the received data, the cumulative number of transmissions in a communication terminal and compared with received counts found that all data has been received, meeting the design requirements.

When the control center requests data from the relay node every fixed period of time, the correct rate of data transmission is limited by communication nodes. When the number of communication nodes are large, the communication between the data relay node and communication nodes will be interrupted by control center's request, thus reducing the accuracy of the data transmission system.

Set the experimental parameters the same as above, the control center request data to the relay node every 5.5s and then, waiting for data reception. Comparing the received data with the sent data, and get the correct rate of data transmission, as shown in Figure 5. Thus, when the system of communication nodes is within a certain amount , the communication of the whole system will be maintained in good effect.

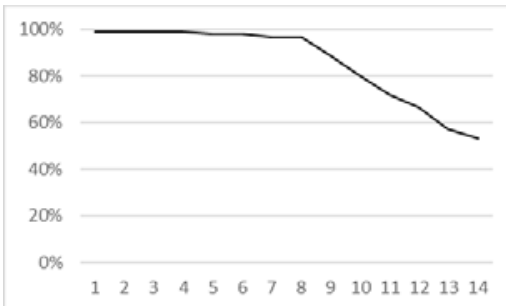

Fig. 5 The relationship between data accuracy and the number of nodes

\section{Conclusion}

The system adapts TDMA communication mechanism to establish a multi-level communication network without base stations. In the system we defined communication protocol and communicate through micro-power wireless data module YL-500. Its advantages include low cost and mature wireless communication technologies, which make this system easily achieved. And its stable work and reliable transmission have been validated by a specific test. Also the establishment of network doesn't need the base stations. In addition, it is convenient for this system to extend when the software is on the basis of B/S structure. Nonetheless, there are many issues worthy of further study such as the data security.

\section{References}

[1] Cui Hu. Implications for foreign coal mine safety management of domestic mine, J. Coal Mine Modernization, 2005 (2):32-33.

[2] Cuihai Liu, Dong Weng, Simulation and Application of Radio Communication System, National Defense Industry Press, Beijing, 2013.

[3] Mingzhu Chen, The basis of software radio, Higher Education Press, Beijing, 2008.

[4] George F. Elmasry, Tactical Wireless Communications and Networks Design Concepts and Challenges, National Defense Industry Press, Beijing, 2014.

[5] Ge songhu. TDMA-based MAC protocol design and implementation of cooperative. National University of Defense Technology, 2011.

[6] Information on http:/www.globalsecurity.org/militart/system/ground/internet-t.htm 